\title{
Shaping up two-photon imaging in vivo
}

\begin{abstract}
A new module, easily adapted to most two-photon imaging systems, enables video-rate volumetric imaging with synaptic resolution
\end{abstract}

In conventional two-photon brain imaging, imaging quickly typically means having to move the focus of the laser rapidly, relying on devices like acousto-optic lenses. While this approach works, and has produced impressive data on populationlevel neural dynamics, it comes with some drawbacks. For example, by continuously taking 3-D image stacks, you build up very large data sets that can be difficult to analyze. Likewise, motions of the brain that move neurons in and out of the laser's focal plane can lead to loss of information. To help solve these problems with volumetric two-photon imaging in vivo, $\mathrm{Na} \mathrm{Ji}$ at the Janelia Research Campus, Howard Hughes Medical Institute, Virginia, and colleagues have developed a new imaging module that easily adapts to most two-photon microscopes and provides fast, high-resolution volumetric imaging of neural activity (Nat. Neurosci. 20, 620-628; 2017).

"The push in neurobiology right now is to image the activity of neurons throughout as large a volume as possible and with as high a speed as possible," comments Ji. The reason for this, as Ji explains, is that everything in the brain is spread out in three dimensions: neurons extend processes to deliver and receive information with other neurons, and networks of neurons can expand hundreds of microns, if not wider, throughout the nervous system. For opaque brains, like in humans and mice, two-photon calcium imaging is a great tool, allowing neuroscientists to penetrate relatively deep into the scattering tissue of areas like the neocortex. But capturing in vivo neural activity in a large volume of tissue with high temporal and spatial resolution is still difficult.

"The challenge is how can you do twophoton imaging really quickly, while still keeping the resolution very high," notes Ji. "Our new module is one approach to solve this problem."

Rather than constantly refocusing the laser beam of the two-photon microscope to build image stacks, their module stretches out the focus, forming a 'Bessel beam', which extends the depth of view in the $z$-direction. The process is not entirely dissimilar to using a low numerical aperture objective, which as most microscope users know, can help increase the range of what you can clearly visualize in the $z$-plane. But, there's a reason why these objectives typically cost a fraction of the price of ones with higher numerical apertures; while the $z$-range is improved, the resolution in the $x-y$ planes is significantly diminished, eliminating the ability to image small processes.

The Bessel beams generated by the Ji lab's module does not come at such a cost; as they demonstrate in their paper, extended $z$-ranges can be produced while maintaining high lateral resolution, all with video-rate speeds to capture rapid network dynamics.

The Ji lab also showed off their module's impressive flexibility, fitting it to three different two-photon microscopes (one commercial scope, and two custom-made), and imaging in vivo neural activity in a range of samples, including fruit flies, zebrafish larvae, mice and ferrets.

As Ji puts it, "some technology developers want to do the the most technically challenging experiments and say, 'look what I can do'. But then nobody ever uses the technology because it's too hard to adopt." Ji's goal with the Bessel module was to develop something that is easy for
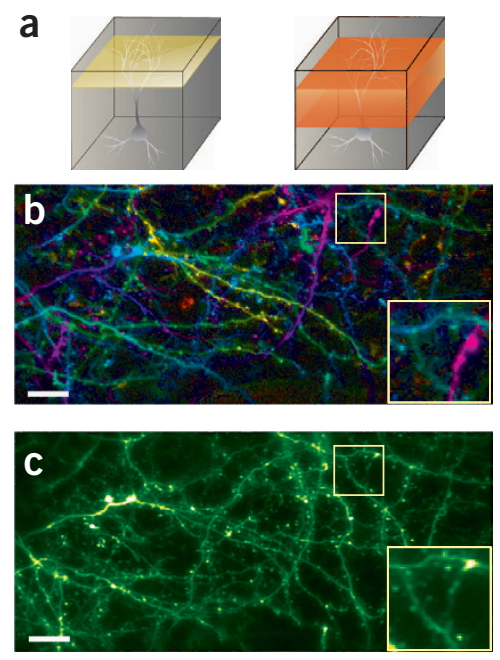

Imaging synapses using Bessel module.

(a) Schematic demonstrating the extended $z$-range focus using the Bessel module, and example two-photon calcium images using (b) conventional focusing and (c) Bessel focusing. Adapted from Nat. Neurosci. 20, 620-628; 2017.

others to adopt and apply in a variety of experimental settings. "I really want people to use it, and I think the only way you can convince someone is to demonstrate that the technique is easy to adopt and is useful across a wide range of samples."

Ji notes that the current module has some limitations: to avoid confusion, only sparsely labeled neurons can be accurately monitored. But, her lab has plans to overcome this limitation. She is currently collaborating with Liam Paninski's and Darcy Peterka's labs at Columbia University to develop analysis methods that would enable densely labeled and overlapping neurons to be accurately separated and tracked, potentially opening up the Bessel module to address new questions in population-level neural activity in awake-behaving animals. Dustin M. Graham 